\title{
DESIGN OF FUZZY RULE-BASED CLASSIFIERS THROUGH GRANULATION AND CONSOLIDATION
}

\author{
Andri Riid, Jürgo-Sören Preden \\ Laboratory for Proactive Technologies, Tallinn University of Technology, \\ Ehitajate tee 5, 19086, Tallinn, Estonia
}

\begin{abstract}
This paper addresses the issue how to strike a good balance between accuracy and compactness in classification systems - still an important question in machine learning and data mining. The fuzzy rule-based classification approach proposed in current paper exploits the method of rule granulation for error reduction and the method of rule consolidation for complexity reduction. The cooperative nature of those methods - the rules are split in a way that makes efficient rule consolidation feasible and rule consolidation itself is capable of further error reduction - is demonstrated in a number of experiments with nine benchmark classification problems. Further complexity reduction, if necessary, is provided by rule compression.
\end{abstract}

Keywords: pattern recognition, fuzzy classification, complexity reduction

\section{Introduction}

Classification is a basic task in performing data analysis or pattern recognition, therefore many problems in very different fields (such as biology, medicine, information retrieval, national security, speech/handwriting recognition, spam filtering etc.) can be represented as classification problems. This explains the need for increasingly accurate, reliable and computationally efficient classifiers [3].

Simply put, a classifier is a function that assigns a class label to an object (observation) on the basis the object description. The latter is given by a vector that contains values of the features or attributes (present paper focuses only on data sets with numerical attributes but the attributes can also be nominal and ordinal) of the object that are considered to be relevant for the classification task. Usually, the classifier is trained to predict class labels using a training algorithm and a training data set. Once the training is completed, the classifier is expected to perform favorably on unseen objects.
There exists a number of classification algorithms including Bayesian classifiers [12], nearest neighbor classifiers [11], rule-based classifiers [9], support vector machines [10], classification trees $[6,26]$, neural classifiers [8, 23], fuzzy logic-based classifiers $[4,17,19]$ and many hybrid and ensemble methods $[27,30]$.

Fuzzy rule-based classifiers are fuzzy systems specifically configured for performing classification tasks that consist of a number of classification rules and utilize fuzziness only in the reasoning mechanism of the classifier [21]. Often, fuzzy rule-based classifiers are considered more intuitive and interpretable than more common black box classification systems [24]. Although interpretability and accuracy are considered to be contradictive requirements, fuzzy rule-based classifiers are not necessarily less accurate than other classifiers.

Generally speaking, the goal in fuzzy rulebased classification is to obtain the maximum possible classification accuracy with as simple classifier as possible. Classification accuracy that a data- 
driven fuzzy rule-based classifier is able to achieve, first and foremost depends on the properties of the data set. Instances of classes that are separate from instances of other classes in product space are easy to classify correctly whereas high overlap of classes can make it very difficult to obtain an accurate classifier.

The class distributions that do not separate naturally in product space typically need to be modeled with increased level of granularity. Alternatively, optimal or near-optimal decision border may be provided by suitable rule placement. In this study we propose an approach that explores both these options to yield accurate yet compact classifiers.

The proposed approach includes the following steps: 1) classifier initialization (generation of a minimal rule classifier); 2) rule splitting procedure that gradually increases granularity of the classifier until satisfying accuracy level has been met; 3 ) rule consolidation that reduces the number of classification rules either maintaining the obtained level of accuracy or even improving on that. These steps of the approach are described in sections 3.1, 3.2 and 3.3 , respectively.

The 10-fold cross-validation classification results on nine benchmark datasets provided in section 5 demonstrate the competitiveness of the proposed approach. If desired, complexity of a fuzzy classifier can be further reduced by reducing the number of conditions in the rules (termed as rule compression).

\section{Preliminaries}

A fuzzy rule-based classifier consists of $R$ rules in the following format

$$
\begin{gathered}
\text { IF } x_{1} \text { is } A_{1 r} \text { AND } x_{2} \text { is } A_{2 r} \text { AND } \ldots \text { AND } \\
x_{N} \text { is } A_{N r} \text { THEN } y \text { belongs to class } c_{r},
\end{gathered}
$$

where $A_{i r}$ denote the linguistic labels of the $i$-th feature associated with the $r$-th rule $(i=1, \ldots, N ; r=$ $1, \ldots, R)$ and $c_{r}$ is a class label assigned to the $r$-th rule $\left(c_{r} \in\{1, \ldots, T\}\right)$. Note that the actual numerical value of $c_{r}$ is irrelevant, it just functions as a label because class is a nominal variable.

Each $A_{i r}$ has its representation in the numerical domain - a typically normal and convex mem- bership function $\mu_{i r}$. In present study we employ the membership functions (MFs) that are built upon two Gaussian curves defined by the positions of the peaks $b_{1}$ and $b_{2}$ and standard deviations $\sigma_{1}$ and $\sigma_{2}$, respectively. We assume that $b_{1}=b_{2}=b$ thus the MF appears as

$$
\mu(x)=\left\{\begin{array}{cc}
e^{-\frac{(x-b)^{2}}{2 \sigma_{1}^{2}}}, \quad x<b \\
e^{-\frac{(x-b)^{2}}{2 \sigma_{2}^{2}}}, \quad x \geq b
\end{array} .\right.
$$

From 2 we derive the expressions for $\sigma_{1}$ and $\sigma_{2}$ so that $\mu(a)=\mu(c)=\alpha, \alpha<1$.

$$
\begin{aligned}
& \alpha=e^{-\frac{(a-b)^{2}}{2 \sigma_{1}^{2}}} \Rightarrow \sigma_{1}=\sqrt{-\frac{(a-b)^{2}}{2 \ln (\alpha)}}, \\
& \alpha=e^{-\frac{(c-b)^{2}}{2 \sigma_{2}^{2}}} \Rightarrow \sigma_{2}=\sqrt{-\frac{(c-b)^{2}}{2 \ln (\alpha)}} .
\end{aligned}
$$

By substituting 3 and 4 into 2 we obtain

$$
\mu(x)=\left\{\begin{array}{ll}
e^{\frac{\ln \alpha(x-b)^{2}}{(a-b)^{2}}}, & x<b \\
e^{\frac{\ln \alpha(x-b)^{2}}{(c-b)^{2}}}, & x \geq b
\end{array},\right.
$$

which further simplifies into

$$
\mu(x)=\left\{\begin{array}{ll}
\alpha^{\left(\frac{x-b}{a-b}\right)^{2}}, & x<b \\
\alpha^{\left(\frac{x-b}{c-b}\right)^{2}}, & x \geq b
\end{array} .\right.
$$

Note that the parameters of $6-a, b, c$ and $\alpha-$ are easier to interpret than the standard deviations of 2 that is evidenced in Figure 1.

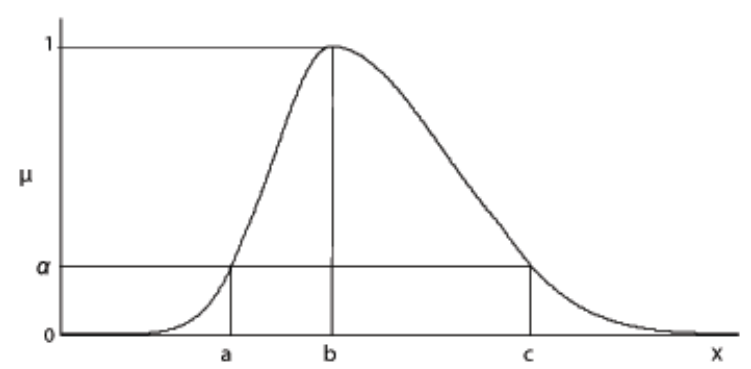

Figure 1. The Gaussian MF employed in current study and the meaning of its parameters.

The reasoning mechanism of a fuzzy rule-based classifier is usually implemented by the single winner approach $[1,16,18,20,24,31]$ that selects the class label $c_{r}$, associated with the rule that provides the highest rule activation degree $\left(\tau_{r}\right)$ for the vector 
$\mathbf{x}_{k}=\left(x_{1}(k), x_{i}(k), \ldots, x_{N}(k)\right)$ representing $k$-th observation.

$$
y(k)=c_{r}, \underset{1 \leq r \leq R}{\arg \max }\left(\tau_{r}(k)\right),
$$

where

$$
\tau_{r}(k)=\bigcap_{i=1}^{N} \mu_{i r}\left(x_{i}(k)\right),
$$

where $\bigcap_{i}^{N}$ represents the minimum operator corresponding to the linguistic operator AND in 1.

\section{The proposed method}

This Section starts with the description of the rule generation routine that is applied whenever a subset of data is at hand, upon which a classification rule needs to be constructed.

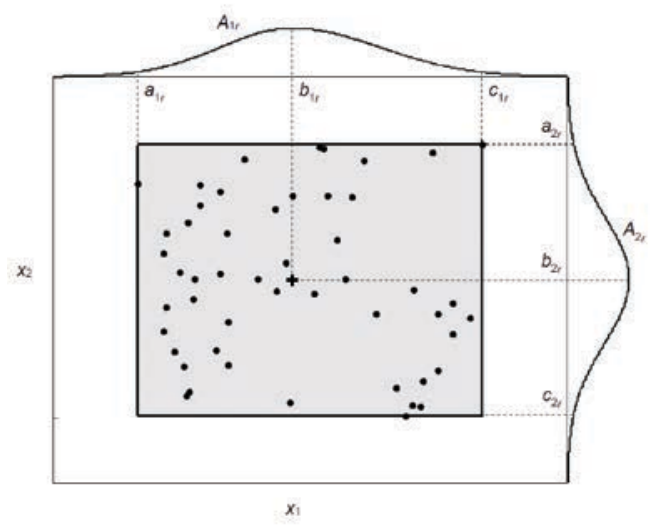

Figure 2. Generation of the $r$-th rule in product space $(N=2)$. Note that within the rule borders (grey area) $\tau_{r}>\alpha$ (this is ensured by the minimum operator in refeq:tau).

Given a subset of data $S_{r}$ that contains $K_{r}$ observations and its mean $\mathbf{m}_{r}=\left(m_{1 r}, m_{2 r}, \ldots, m_{N r}\right)$ that is the geometric centroid of the data points in $S_{r}$

$$
\mathbf{m}_{r}=\sum_{k \in S_{r}} \mathbf{x}_{k} / K_{r}
$$

the MFs $\mu_{i r}$ of form refeq:mfs are created in all dimensions $i$. Given a predefined value of $\alpha(\alpha=$ 0.005 in all following experiments), the MF parameters $a_{i r}, b_{i r}, c_{i r}$ are obtained as follows. For each $i$

$$
\begin{gathered}
a_{i r}=\min _{k \in S_{r}}\left(x_{i}(k)\right), c_{i r}=\max _{k \in S_{r}}\left(x_{i}(k)\right), \\
b_{i r}=m_{i r} .
\end{gathered}
$$

Following this a rule

$$
\begin{aligned}
& \text { IF } x_{1} \text { is } A_{1 r} \text { AND } x_{2} \text { is } A_{2 r} \ldots \\
& \text { AND } x_{i} \text { is } A_{i r} \ldots \text { AND } x_{N} \text { is } A_{N r} \\
& \text { THEN } y \text { belongs to class } c_{r},
\end{aligned}
$$

where $A_{i r}$ represent the MFs $\mu_{i r}$ and $c_{r}$ is the class that is represented by the majority of observations in subset $S_{r}$, is constructed (Figure 2).

\subsection{Minimal rule classifiers}

The simplest classifier possible is the minimal rule classifier (MRC) that specifies only one rule for each class. The training data set is divided into $T$ subsets so that each subset contains only the samples belonging to one of $T$ classes and the rule generation routine refeq:makeMFs-refeq:classst 1 is executed until all subsets have been covered. Unless the classes are well separable in the product space, the MRC usually comes with a number of misclassified samples, depending on how "bad" the data is.

It is worth noting that if we replace the designated MFs refeq:mfs with standard Gaussian functions

$$
\mu(x)=\frac{1}{\sigma \sqrt{2 \pi}} e^{-\frac{(x-b)^{2}}{2 \sigma^{2}}}
$$

and use

$$
b_{i r}=m_{i r}, \sigma_{i r}=\sqrt{\frac{1}{K_{r}} \sum_{k \in S_{r}}\left(x_{i}(k)-m_{i r}\right)^{2}},
$$

in the rule generation process and multiply $\tau_{r}(k)$ in refeq:classinf by a rule weight $w_{r}(k)$, which is computed as the prior probability of class $c_{r}$ samples in training data (the number of samples belonging to class $c_{r}$ divided by the overall number of samples $K$ ) then the MRC what we obtain is, in fact, a Naive Bayesian classifier.

\subsection{Rule granulation for error minimiza- tion}

Each rule of the minimal rule classifier governs a subset of data $S_{r}$ consisting of $K_{r}$ samples and usually there is a number of misclassified samples within this subset. The latter figure is denoted by $\eta_{r}$ and called local error. The global error $(\eta)$ is given by

$$
\eta=\sum_{r=1}^{R} \eta_{r}
$$


Classification error reduction is carried out by a sequence of rule splits so that at each iteration a parent rule is selected and split into two offspring rules. The offspring rules replace the parent rule, which means that at each iteration the number of classification rules increases by one. Usually there is a number of choices on which parent rule to pick and how to make the split. The first choice for the parent rule is a rule $p$ with the highest local error

$$
p=r, \underset{1 \leq r \leq R}{\arg \max }\left(\eta_{r}\right) .
$$

If there are several rules with the same local error, we simply choose the one with the highest $K_{r}$ of those.

The rule splitting cut can be made around each erroneous sample under the parent rule. At given iteration, a single cut is allowed at one of $\mathrm{N}$ coordinates, thus the overall number of potential rule splits at the iteration equals $N \times \eta_{p}$. For example, in the situation depicted in Figure 3, $N=2, \eta_{p}=1$ and thus two cuts are possible.

A cut divides the $K_{p}$ samples of the parent rule into two subsets $S_{o}$ and $S_{q}$ that form the basis of two offspring rules, $\mathrm{R}_{o}$ and $\mathrm{R}_{q}$. Note that the erroneous sample is always sided with the offspring rule that contains less samples. Of available cuts the one that results in the best performing classifier (yielding the least number of $\eta$ ) is selected. It is possible that there are several cuts that result in classifiers with the same number of erroneous samples. In this case we choose the cut that has the minimal value of $\max \left(\eta_{o}, \eta_{q}\right)$ - generally this leads to faster convergence. If this still leaves us several equally good candidates, we choose the cut that has a smaller value of $\min \left(K_{o}, K_{q}\right)$.

The splitting continues until $\eta$ reaches either zero (as in Figure 3, right), some other pre-specified higher value or yet another ending criterion (e.g. pre-set overall accuracy rate, overall number of rules) becomes satisfied.

\subsection{Rule consolidation}

The procedure for reducing the number of rules of classifiers is outlined in [29] and termed rule base consolidation. During the consolidation, weaker rules (governing few samples) are constantly losing their samples to stronger rules (those governing many samples). Each such sample transfer is valid as long as accuracy of the classifier is not compromised. As a natural result, many of the weaker rules become obsolete.

The rules are ranked by their strength (the number of samples they govern) in ascending order $p \in\{1, \ldots, R\}$. The process starts from the lowest ranked rule $(p=1)$ :

1. Pick a rule $\mathrm{R}_{r}$ with the rank $p$.

2. Pick $k$-th sample $\left(k=1, \ldots, K_{r}\right)$ from the subset $S_{r}$ governed by rule $\mathrm{R}_{r}$.

3. Transfer this sample from $S_{r}$ to the subset $S_{q}$ corresponding to $\mathrm{R}_{q}$, the next rule in the ranking that matches the class of the sample $\left(c_{q}=y_{k}\right)$.

4. Update the MFs of both $\mathrm{R}_{r}$ and $\mathrm{R}_{q}$ on the basis of modified subsets $S_{r}$ and $S_{q}$, respectively.

It is then verified if the accuracy loss due to consolidation has occurred (the global error of the consolidated classifier has increased). If there is no accuracy loss, the tranfer is accepted. Otherwise, the transfer is rejected. Based on this decision we proceed as follows:

- If the transfer is accepted and $k<K_{r}$, increment $k$ (select the next sample from $S_{r}$ ). If $k$, however, already equals $K_{r}$, delete rule $\mathrm{R}_{r}$ along with associated MFs, update the ranking, increment $p$ and go back to step 1 .

- If the transfer is rejected, first discard the changes to the MFs of $\mathrm{R}_{r}$ and $\mathrm{R}_{q}$, pick the next matching rule from the ranking and go back to step 3. If we already have reached the last matching rule in the ranking, select the next sample from subset $S_{r}$ (increment $k$ ) and go to step 2. If $k$ already equals $K_{r}$ as well, increment $p$ and return to step 1 .

The process comes to an end when we have reached the last rule in the ranking $(p=R)$. It can be, however, started over from the beginning and carried on until the consolidation stabilizes (i.e. there are no more accepted transfers).

\section{Results}

All nine data sets that have been chosen for classification experiments and verification of the pro- 

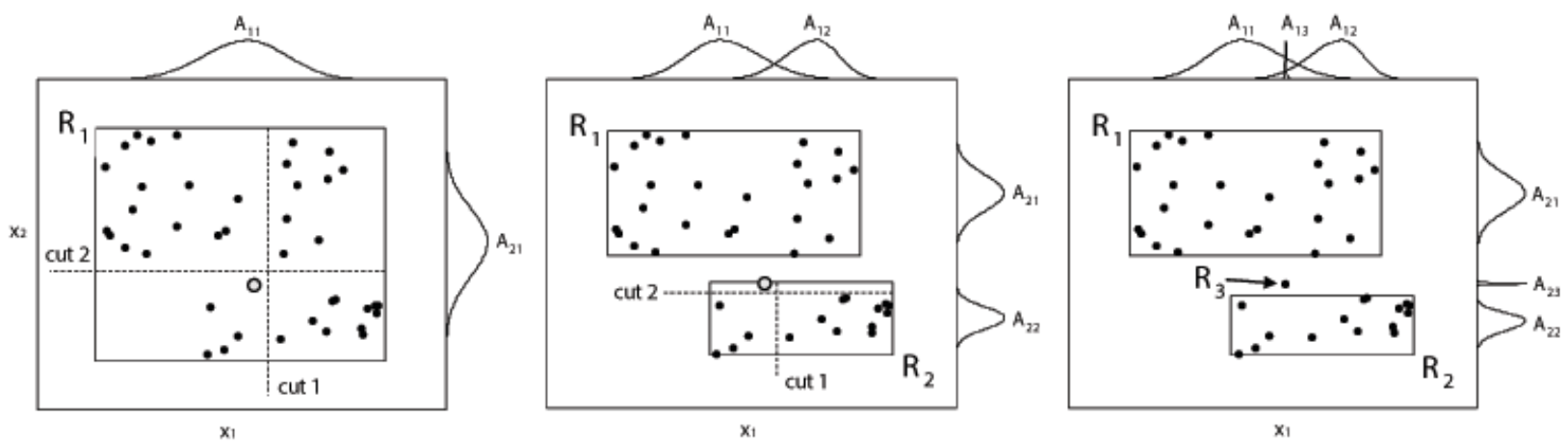

Figure 3. Rule granulation. With other criteria being equal, cut 2 in the left graph is preferred over cut 1 because the offspring rule $\mathrm{R}_{2}$ governs less samples than any of involved rules in the alternative scenario. Cut 2 in the middle graph is preferred over cut 1 because it promptly reduces the classification error to zero.

posed method, are well-known, feature frequently in classification and pattern recognition studies and are available through the UCI Machine Learning Repository [5]. These are the Iris [14], Wine [2], Thyroid [25], Glass [13], BUPA Liver Disorders [34], Cleveland Heart Disease [15], Pima Indian Diabetes [32] and two variants of Wisconsin Breast Cancer data sets (referred to as WDBC [33] and Breast Cancer [35], respectively).

The MRCs for those classification problems are given in Table 1.

Table 1. MRC results on benchmark problems.

\begin{tabular}{c|c|c|c|c|c}
\hline Data set name & $N$ & $T$ & $K$ & $\varepsilon(\%)$ & $\eta$ \\
\hline Iris & 4 & 3 & 150 & 94.67 & 8 \\
Wine & 13 & 3 & 178 & 95.51 & 8 \\
Thyroid & 21 & 3 & 3772 & 99.89 & 4 \\
WDBC & 30 & 2 & 569 & 95.61 & 25 \\
Breast Cancer & 9 & 2 & 683 & 58.27 & 285 \\
Glass & 9 & 6 & 214 & 58.88 & 88 \\
Bupa & 6 & 2 & 345 & 59.13 & 141 \\
Cleveland & 13 & 5 & 297 & 24.58 & 224 \\
Pima & 8 & 2 & 768 & 55.99 & 338 \\
\hline
\end{tabular}

The table shows the number of features $(N)$, the number of classes $(T)$ and the number of samples $(K)$ for each data set, as well as the number of misclassified samples $(\eta)$ and overall accuracy $(\varepsilon)$ of corresponding MRCs. The latter is computed as

$$
\varepsilon=1-\eta / K,
$$

and we can see that for several classification problems listed in Table 1, accuracy of MRCs is not particularly high.
Table 2. Comparison of simple classifiers on benchmark problems.

\begin{tabular}{c|c|c|c|c}
\hline Data set name & $T$ & MRC & NBC & CART \\
\hline Iris & 3 & 96.00 & 96.00 & 96.00 \\
Wine & 3 & 95.51 & $\mathbf{9 8 . 8 8}$ & 88.76 \\
Thyroid & 3 & $\mathbf{9 9 . 8 9}$ & 92.57 & 97.91 \\
WDBC & 2 & $\mathbf{9 5 . 6 1}$ & 94.02 & 92.27 \\
Breast Cancer & 2 & 94.28 & $\mathbf{9 6 . 3 4}$ & 92.68 \\
Glass & 6 & $\mathbf{7 2 . 4 3}$ & 54.67 & 71.03 \\
Bupa & 2 & $\mathbf{6 5 . 8 0}$ & 55.94 & 63.19 \\
Cleveland & 5 & 59.93 & $\mathbf{6 2 . 2 9}$ & 59.60 \\
Pima & 2 & 67.19 & $\mathbf{7 6 . 1 7}$ & 73.57 \\
\hline
\end{tabular}

Rule consolidation algorithm alone is often able to improve the classification accuracy because it can transfer the misclassified samples to properly labelled rules. For example, when rule consolidation is applied to the MRCs from Table 1, it reduces the initial classification error considerably for some, previously ill-classified data sets. This way, classification accuracy for the Iris data set increases from $94.67 \%$ to $96.00 \%$ (6 erroneous samples); from $58.27 \%$ to $94.29 \%$ (39 erroneous samples) for Breast Cancer data set; from 59.13\% to $65.80 \%$ (118 erroneous samples) for BUPA data set; from $24.58 \%$ to $59.93 \%$ (119 erroneous samples) for Cleveland Heart Disease data set; from $55.99 \%$ to $67.19 \%$ (252 erroneous samples) for Pima Indian Diabetes data set and from 58.88\% to $72.43 \%$ (59 erroneous samples) for the Glass data set.

Comparison between the consolidated MRCs, Naive Bayesian classifiers and decision trees (CART, [6]) with $T$ leaf nodes is given in Table 2. The best result for each data set is highlighted. 
Figure 4 plots the error curves in blue throughout the rule granulation steps for all benchmark data sets. Depending on the initial error and data set properties it takes a varying number of splits to obtain the minimum error classifier (MEC) at the last split. Broadly speaking, the data sets in the first row are the easy ones, the data sets in the second row are more difficult and the ones in the last row present a challenge. Typically, we can see a rapid improvement of classification accuracy in the beginning of the training process (which does not last long). This is followed by a more hectic or stagnation phase where there is almost no improvement (the latter phenomenon is particularly clearly evidenced in the graph related to the Breast Cancer data set) just further fragmentation of rules. In the last phase of the training, however, the improvement is slow but steady (this is because at this point the rules that contain errors are small).

Table 3. the number of rules of benchmark data set MECs before $\left(R_{s}\right)$ and after consolidation $\left(R_{c}\right)$ and the number of leaf nodes of decision trees describing the same problem $\left(n_{l}\right)$.

\begin{tabular}{c|c|c|c}
\hline Data set name & $R_{s}$ & $R_{c}$ & $n_{l}$ (CART) \\
\hline Iris & 10 & 7 & 9 \\
Wine & 9 & 4 & 12 \\
Thyroid & 10 & 6 & 13 \\
WDBC & 24 & 7 & 22 \\
Breast Cancer & 40 & 12 & 32 \\
Glass & 51 & 22 & 50 \\
Bupa & 104 & 35 & 80 \\
Cleveland & 124 & 42 & 101 \\
Pima & 202 & 54 & 128 \\
\hline
\end{tabular}

Table 3 contains the number of rules of MECs after rule granulation $\left(R_{S}\right)$ and after consolidation $\left(R_{c}\right)$. Comparable complexity measure, i.e. the number of leaf nodes $\left(n_{l}\right)$ of a CART applied for the same classification problem is added for reference. From this comparison we can see that while for some data sets $n_{l}$ can be smaller than $R_{s}$, it is always larger than $R_{c}$.

The green curve in Figure 4 indicates the corresponding accuracy rates of the classifiers to which instantaneous consolidation is applied after each split. We can see that when rule consolidation is applied to an erroneous classifier it typically gives a significant boost in accuracy, especially for "dif- ficult" data sets. The red curve tied to the second $y$-axis in these graphs depicts the number of rules after each consolidation operation.

In practice, however, we do not need so much a flawless and possibly overtrained classifier with many parameters, rather than a compact one that would capture the essence of the classification problem. For this we should be able to guess the breaking point or the "soft spot" in learning located somewhere in the second phase when the algorithm is turning its attention to the erroneous samples, which, for all we know could be just measurement errors or outliers in the data.

\section{Performance on unseen data}

In previous Sections we have shown that using the combination of rule granulation and consolidation, it is possible to obtain the classifiers of arbitrary accuracy (on training data, that is). In practice, however, the ability of a classifier to learn the training data is less important than its generalization ability, i.e its ability to predict the class labels for new, unseen samples of data. This ability is usually estimated using 10 -fold cross validation by which the original data set is randomly divided into 10 disjoint sets (folds) of equal size where each fold has roughly the same class distribution. The classifier is trained 10 times, each time with a different set held out as a test set and the other 9 subsets put together to form a training set. This way each data point gets to be in a test set exactly once and in a training set 9 times. In the end, the mean values of training and testing accuracies across all 10 trials are computed that serve as the performance measures.

We have performed cross validation on four types of classifiers, namely: Naive Bayesian classifiers, consolidated MRCs, classifiers obtained with the proposed granulation-consolidation method and CARTs.

Note that in order to avoid overfitting in the proposed method, the rules are split until the highest local error has come down to a pre-specified value $\eta_{\text {min }}$ that is roughly correlated to the initial training error. For Iris, Wine and Thyroid data sets, thus $\eta_{\min }=1$, for WDBC, Breast Cancer and Glass data sets, $\eta_{\min }=3$, for BUPA and Cleveland data sets $\eta_{\text {min }}=5$ and for the Pima data set $\eta_{\text {min }}=10$. The 

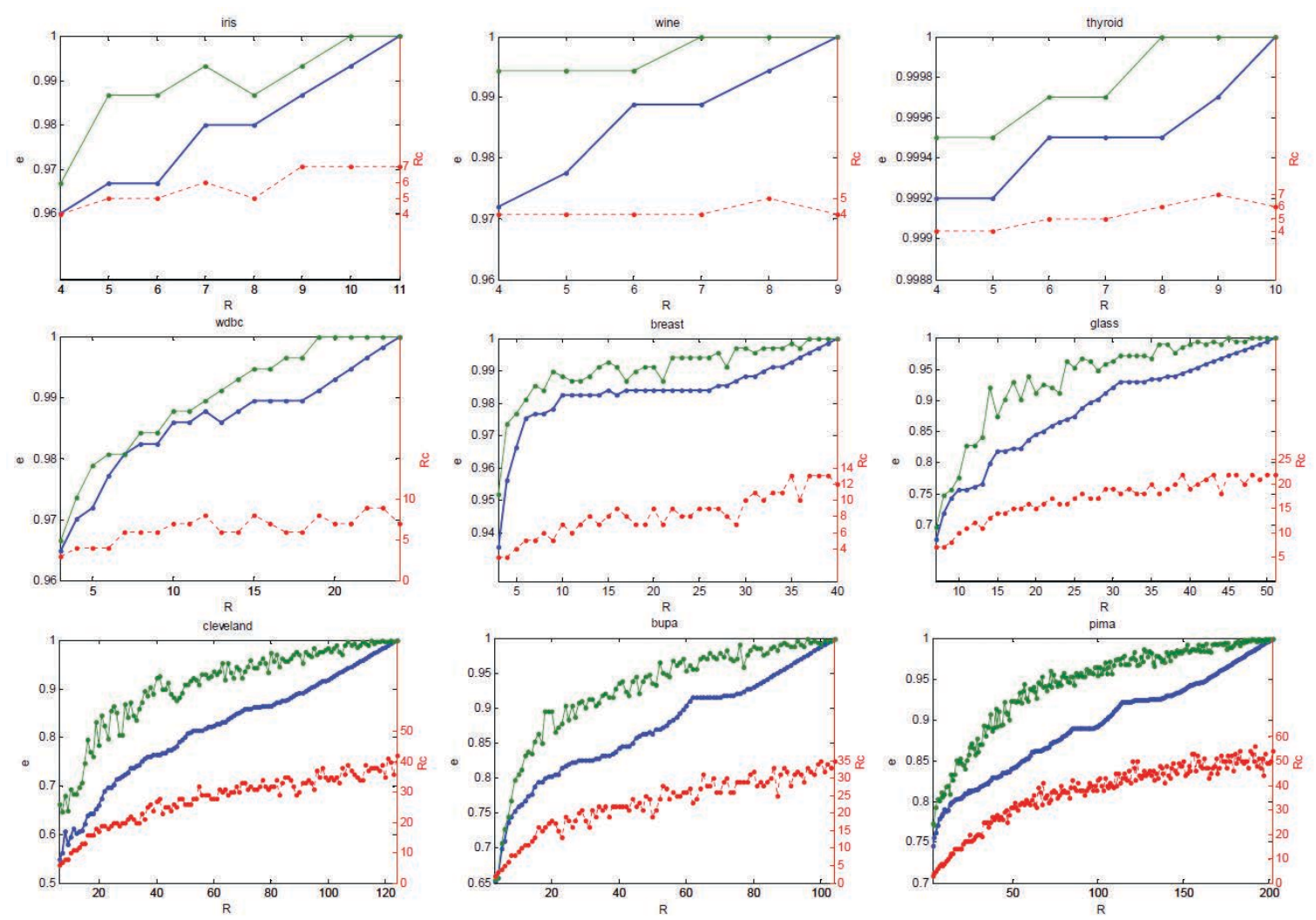

Figure 4. The learning curves of the proposed approach on nine benchmark data sets with and without rule consolidation (green and blue lines, respectively). The number of consolidated rules at each training step is depicted by a red curve.

Table 4. Stratified 10-fold cross validation results on benchmark data sets.

\begin{tabular}{|c|c|c|c|c|c|c|c|c|c|c|c|c|}
\hline \multirow{2}{*}{ Data set } & \multicolumn{3}{|c|}{ NBC } & \multicolumn{2}{|c|}{ MRC } & \multicolumn{4}{|c|}{ proposed method } & \multicolumn{3}{|c|}{ CART } \\
\hline & $R$ & $\varepsilon_{t r}$ & $\varepsilon_{t s t}$ & $\varepsilon_{t r}$ & $\varepsilon_{t s t}$ & $R_{S}$ & $R_{C}$ & $\varepsilon_{t r}$ & $\varepsilon_{t s t}$ & $n_{l}$ & $\varepsilon_{t r}$ & $\varepsilon_{t s t}$ \\
\hline Iris & 3 & 96.07 & 95.33 & 95.85 & 94.67 & 5.5 & 4.4 & 98.30 & 96.67 & 4.7 & 98.15 & 95.33 \\
\hline Wine & 3 & 98.44 & 96.66 & 95.88 & 91.63 & 5.2 & 4.0 & 99.62 & 95.00 & 8.1 & 99.19 & 90.52 \\
\hline Thyroid & 3 & 92.59 & 92.67 & 99.81 & 99.42 & 5.3 & 4.0 & 99.94 & 99.44 & 11.2 & 99.98 & 99.66 \\
\hline WDBC & 2 & 93.91 & 93.33 & 95.72 & 93.85 & 8.4 & 5.5 & 98.63 & 94.03 & 8.3 & 97.85 & 93.68 \\
\hline $\mathrm{BC}$ & 2 & 96.24 & 96.20 & 92.24 & 92.39 & 8.5 & 5.7 & 98.75 & 95.32 & 9.6 & 97.69 & 95.31 \\
\hline Glass & 6 & 54.41 & 50.87 & 68.70 & 53.75 & 20.1 & 13.9 & 93.82 & 65.46 & 19.2 & 87.07 & 62.73 \\
\hline BUPA & 2 & 57.46 & 54.23 & 64.38 & 59.50 & 22.9 & 16.1 & 90.60 & 69.56 & 19.5 & 84.35 & 68.96 \\
\hline Cleveland & 5 & 63.63 & 55.13 & 61.58 & 53.98 & 29.9 & 18.8 & 86.04 & 54.86 & 26.4 & 78.04 & 53.22 \\
\hline Pima & 2 & 76.45 & 75.26 & 69.46 & 67.97 & 27.3 & 19.8 & 89.19 & 75.26 & 22.5 & 85.32 & 73.83 \\
\hline
\end{tabular}


same values of $\eta_{\min }$ serve as the node splitting stop criteria for individual data sets in a CART - a node is no longer split if it contains $\eta_{\min }$ (or less) erroneous samples. Also note that the data folds are fixed for all compared algorithms to obtain comparable classification results.

The results of the validation are given in Table 4 where $\varepsilon_{t r}$ and $\varepsilon_{t s t}$ denote the training and testing accuracies, respectively, and the best testing accuracy for each data set is highlighted.

The cross-validation results partially confirm the frequent claim that "Naive Bayes can often outperform more sophisticated classification methods" [22] as Naive Bayes appears to be winner in 3 out of 9 cases and ties once with the proposed method. When it loses, however, it can lose by a large margin as the results on Glass, BUPA and Thyroid data sets indicate.

On the other hand, the proposed method is the overall winner in still more cases, outperforms CART in terms of testing accuracy in 8 out of 9 cases and the number of fuzzy rules after consolidation $\left(R_{c}\right)$ is always smaller than the corresponding number of leaf nodes $\left(n_{l}\right)$. By definition, however, the attribute tests are applied only to a handful of available attributes in the internal nodes that are in the path from the root node to a leaf node in a CART, which would correspond to a fuzzy rule having a limited number of conditions (so called incomplete rules).

To reduce the number of conditions in fuzzy classifiers we apply the naive rule compression method [28]. The algorithm is based on simple trial and error and is described as follows:

1. Pick the rule $\mathrm{R}_{r}(r=1, \ldots, R)$.

2. Rank the features $i=1, \ldots, N$ by MF spread $\left(c_{i r}-a_{i r}\right)$, normalized, of course, in descending order (this way the features in which the subset of samples governed by $r$-th rule is less compact, less defined, are removed first).

3. Discard the conditions applied to the features one by one, in the order of ranking, reversing those removals that would result in loss of accuracy.

The average numbers of conditions before and after compression - $n_{\text {cond }}$ and $n_{\text {cond }}^{*}$, respectively - of fuzzy classifiers are given in Table 5 along with cross-validated classification accuracy before and after compression (values of $\varepsilon_{t s t}$ are lifted from Table 4). The adjusted number of attribute tests, i.e. the total number of internal nodes met on $n_{l}$ individual paths from the root node to all leaf nodes in a CART (tests concerning the same attribute in a given path are taken into account only once) is added for reference. One can see that the number of conditions is below the number of attribute tests but this is partially so because $n_{l}$ tends to be larger than $R_{c}$. The average number of conditions per rule in a classifier, however, is only $10 \%$ higher than the average number of attribute tests per leaf node, which is good enough.

Fuzzy rules with less conditions appear to be more general, and thus one would expect them to have more generalizational power, however, as our results indicate, this is true only for half the cases.

To put this piece of research into context, one can note that the obtained testing accuracies are in the same ballpark with figures available from literature. For example, [7] that lists the results of various algorithms (Support Vector Machines, associative rules, Naive Bayesian classifiers and decision trees) reports the testing accuracies of $94.5 \pm 2.2 \%$ for Iris, $96.45 \pm 1.35 \%$ for Breast Cancer, $65.95 \pm 5.05 \%$ for BUPA and $75.55 \pm 2.65 \%$ for Pima data sets. In a more recent study [4] with the results of four genetic fuzzy classifiers, four associative classifiers and one decision tree (C4.5), the corresponding figures are $94.65 \pm 1.35 \%$ for Iris, $93.08 \pm 1.62 \%$ for Wine, $74.08 \pm 1.58 \%$ for Pima, 52.86 $\pm 4.04 \%$ for Cleveland, $92.96 \pm 2.29 \%$ for WDBC and $64.65 \pm 6.65 \%$ for Glass data sets.

\section{Conclusions}

The primary goals in fuzzy rule-based classification are accuracy maximization and complexity minimization, which are contradicting requirements. The approach presented in current paper - a combination of rule granulation and rule consolidation methods and further rule compression - offers the possibility to find a good compromise between those requirements.

A properly designed classification algorithm must have good learning abilities to be able to dis- 
Table 5. Cross validation results on compressed classifiers.

\begin{tabular}{c|c|c|c|c|c|c}
\hline \multirow{2}{*}{ Data set name } & \multicolumn{4}{|c|}{ proposed method } & \multicolumn{2}{c}{ CART } \\
\cline { 2 - 7 } & $\varepsilon_{t s t}$ & $\varepsilon_{t s t}^{*}$ & $n_{\text {cond }}$ & $n_{\text {cond }}^{*}$ & $\varepsilon_{\text {tst }}$ & $n_{\text {cond }}$ \\
\hline Iris & 96.67 & $\mathbf{9 7 . 3 3}$ & 17.6 & 8.8 & 95.33 & 8.4 \\
Wine & $\mathbf{9 5 . 0 0}$ & 93.36 & 52.0 & 13.7 & 90.52 & 25.7 \\
Thyroid & 99.44 & 99.55 & 81.9 & 14.6 & $\mathbf{9 9 . 6 6}$ & 50.3 \\
WDBC & $\mathbf{9 4 . 0 3}$ & 93.50 & 165.0 & 25.0 & 93.68 & 25.5 \\
Breast Cancer & 95.32 & $\mathbf{9 5 . 7 6}$ & 51.3 & 22.3 & 95.31 & 29.0 \\
Glass & 65.46 & $\mathbf{6 9 . 5 5}$ & 125.1 & 51.1 & 62.73 & 94.7 \\
BUPA & $\mathbf{6 9 . 5 6}$ & 66.65 & 96.6 & 67.2 & 68.96 & 75.3 \\
Cleveland & 54.86 & $\mathbf{5 6 . 8 8}$ & 244.4 & 100.7 & 53.22 & 127.2 \\
Pima & $\mathbf{7 5 . 2 6}$ & 74.09 & 158.4 & 101.6 & 73.83 & 91.6 \\
\hline
\end{tabular}

cover patterns in data, which is valid for the proposed method but the true criterion of a good classifier is its predictive performance, estimated by cross-validation. However, there is no single classification algorithm that is best for all types of data. A method can outperform others on an almost consistent basis and yet show weaker performance on certain data sets. This is apparent in present study as well as of the chosen benchmarks, the proposed method performs best on Iris, WDBC, Glass, BUPA and Cleveland data sets and ties with Naive Bayesian classifier on Pima data set, whereas Naive Bayesian classifier performs best on Wine and Breast Cancer data sets. CART, on the other hand, outperforms other algorithms on Thyroid data set.

\section{Acknowledgements}

The work presented in this paper was partially supported by the Estonian Ministry of Defence and the European Defense Agency project IN4STARS 2.0.

\section{References}

[1] J. Abonyi, J. A. Roubos, and F. Szeifert, Datadriven generation of compact, accurate, and linguistically sound fuzzy classifiers based on a decisiontree initialization, International Journal of Approximate Reasoning, 23:1-21, 2003

[2] S. Aeberhard, D. Coomans, and O. de Vel, Comparative analysis of statistical pattern recognition methods in high dimensional settings, Pattern Recognition, 27(8):1065-1077, 1994
[3] C. C. Aggarwal, Data Classification: Algorithms and Applications, Chapman \& Hall/CRC, 1st edition, 2014

[4] J. Alcala-Fdez, R. Alcala, and F. Herrera, A fuzzy association rule-based classification model for highdimensional problems with genetic rule selection and lateral tuning, IEEE Transactions on Fuzzy Systems, 19(5):857-872, 2011

[5] K. Bache and M. Lichman, UCI machine learning repository, http://archive.ics.uci.edu/ml, 2013

[6] L. Breiman, J. H. Friedman, R. A. Olshen, and C. J. Stone, Classification and regression trees, Wadsworth \& Brooks/Cole Advanced Books \& Software, Monterey, 1984

[7] B.-C. Chien, J.-Y. Lin, and W.-P. Yang, A classification tree based on discriminant functions, Journal of Information Science and Engineering, 222(3):573594, 2006

[8] S.-B. Cho, Neural-network classifiers for recognizing totally unconstrained handwritten numerals, IEEE Transactions on Neural Networks, 8(1):43-53, 1997

[9] W. W. Cohen, Fast effective rule induction, In Proceedings of the Twelfth International Conference on Machine Learning, pages 115-123, Morgan Kaufmann, 1995

[10] C. Cortes and V. Vapnik, Support-vector networks, Machine Learning, 20(3):273-297, September 1995

[11] T. Cover and P. Hart, Nearest neighbor pattern classification, IEEE Transactions on Information Theory, 13(1):21-27, September 2006

[12] R. Duda and P. Hart, Pattern Classification and Scene Analysis, Wiley, New York, 1973

[13] I. W. Evett and E. J. Spiehler, Rule induction in forensic science, Technical report, Central Research Establishment, Home Office Forensic Science Service, 1987 
[14] R. A. Fisher, The use of multiple measurements in taxonomic problems, Annals of Eugenics, 7(2):179_ 188,1936

[15] J. H. Gennari, P. Langley, and D. Fisher, Models of incremental concept formation, Artificial Intelligence, 40(1-3):11-61, 1989

[16] S. Guillaume and B. Charnomordic, Learning interpretable fuzzy inference systems with FisPro, Information Sciences, 180(20):4409-4427, 2011

[17] J. Hühn and E. Hüllermeier, FURIA: an algorithm for unordered fuzzy rule induction, Data Mining and Knowledge Discovery, 19(3):293-319, 2009

[18] H. Ishibuchi, T. Nakashima, and T. Murata, Three-objective genetic-based machine learning for linguistic rule extraction, Information Sciences, 136(1-4):109-133, 2001

[19] H. Ishibuchi, K. Nozaki, N. Yamamoto, and H. Tanaka, Selecting fuzzy if-then rules for classification problems using genetic algorithms, IEEE Transactions on Fuzzy Systems, 3(3):260-270, 1995

[20] H. Ishibuchi and T. Yamamoto, Rule weight specification in fuzzy rule-based classification systems, IEEE Transactions on Fuzzy Systems, 13(4):428435, 2005

[21] L. Kuncheva, Fuzzy Classifier Design, SpringerVerlag, Heidelberg, 2000

[22] K. Larsen, Generalized naive bayes classifiers, SIGKDD Explorations, 7(1):76-81, 2005

[23] R. P. Lippmann, Neural network classifiers for speech recognition, The Lincoln Laboratory Journal, 1:107-124, 1988

[24] C. Mencar, C. Castiello, R. Cannone, and A. M. Fanelli, Interpretability assessment of fuzzy knowledge bases: A cointension based approach, International Journal of Approximate Reasoning, 52(4):501-518, 2011

[25] J. R. Quinlan, Induction of decision trees, Machine Learning, 1(1):81-106, 1986

[26] J. R. Quinlan, C4.5: Programs for Machine Learning, Morgan Kaufmann Publishers Inc., San Francisco, CA, USA, 1993
[27] Y. Ren, L. Zhang, and P. N. Suganthan, Ensemble classification and regression - recent developments, applications and future directions, IEEE Computational Intelligence Magazine, 11(1):41-53, 2016

[28] A. Riid and J.-S. Preden, Interpretability improvement of fuzzy rule-based classifiers via rule compression, In Proceedings of the 2015 Conference of the International Fuzzy Systems Association and the European Society for Fuzzy Logic and Technology, pages 162-169, Gijon, Spain, 2015

[29] A. Riid and M. Sarv, Determination of regional variants in the versification of estonian folksongs using an interpretable fuzzy rule-based classifier, In Proceedings of the 8th Conference of the $\mathrm{Eu}-$ ropean Society for Fuzzy Logic and Technology (EUSFLAT 2013), pages 61-66, Milan, Italy, 2013

[30] L. Rokach, Pattern Classification Using Ensemble Methods, volume 75 of Series in Machine Perception and Artifical Intelligence, World Scientific Publishing Company, Singapore, 2010

[31] H. Roubos, M. Setnes, and J. Abonyi, Learning fuzzy classification rules from data, Information Sciences, 150(1-2):77-93, 2003

[32] J. W. Smith, J. E. Everhart, W. C. Dickson, W. C. Knowler, and R. S. Johannes, Using the ADAP learning algorithm to forecast the onset of diabetes mellitus, In Proceedings of the Symposium on Computer Applications and Medical Care, pages 261265, Los Alamitos, CA, 1988

[33] W. N. Street, W. H. Wolberg, and O. L. Mangasarian, Nuclear feature extraction for breast tumor diagnosis, In Proceedings of the IS\&T 1993 International Symposium on Electronic Imaging: Science and Technology, volume 1905, pages 861-870, San Jose, CA, 1993

[34] P. D. Turney, Cost-sensitive classification: Empirical evaluation of a hybrid genetic decision tree induction algorithm, Journal of Artificial Intelligence Research, 2:369-409, 1995

[35] W. H Wolberg and O. L. Mangasarian, Multisurface method of pattern separation for medical diagnosis applied to breast cytology, Proceedings of the National Academy of Sciences, 87:9193-9196, 1990 


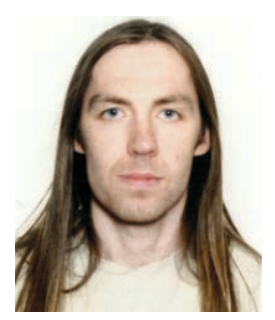

Andri Riid received his M.Sc. and $\mathrm{Ph} . \mathrm{D}$. degrees in System Engineering from Tallinn University of Technology in 1997 and 2002, respectively. He currently works as a Senior Research Scientist in the Laboratory for Proactive Technologies of the same university. His research interests include properties of fuzzy systems and development of algorithms for fuzzy control, modeling and classification.

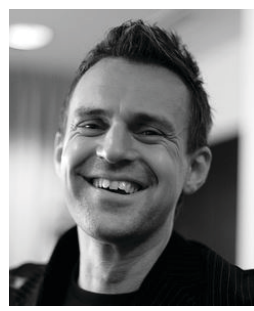

Jürgo-Sören Preden received his Ph.D. degree in System Engineering from Tallinn University of Technology in 2010. He is currently a Senior Research Scientist and Head of the Research Laboratory for Proactive Technologies at Tallinn University of Technology and CEO of Thinnect. His research interests include distributed computing systems, specifically the situation awareness of such systems. He is a member of IEEE. 